OPTICAL PHYSICS

\section{Solitons divide and conquer}

\section{An experimental technique allows packets of light called solitons to maintain their shape in all three dimensions as they travel through a material. Such wave packets could find applications in optical information processing.}

\section{FRANK W. WISE}

$\mathrm{W}$ aves spread out as they propagate. A familiar example is the broadening of a beam of light. The challenge of overcoming the ubiquitous spreading of waves has motivated scientists for decades, and packets of light waves that retain their shape, known as solitons, have been demonstrated in one and two dimensions ${ }^{1}$. However, it has been extremely difficult to create solitons that are stable in three dimensions. Writing in Physical Review X, Lahav et al. ${ }^{2}$ report an experimental approach that can produce such objects. of $3 \mathrm{D}$ solitons to be investigated, and could lead to $3 \mathrm{D}$ solitons that have technological applications.

A narrow beam of light contains wave components that propagate in different directions. As the beam travels through a material, these wave components get out of sync, causing the beam to spread out - a process known as diffraction. However, if the beam is powerful enough, the light changes the material's refractive index (a quantity that describes how light propagates in a medium), which in turn affects the beam. In particular, if the beam has a bell-shaped intensity profile, as do most laser beams, the material focuses the beam like a lens. By tuning the beam intensity, this focusing can counteract diffraction to produce a 'self-guided' beam that does not spread out.

In addition to diffraction, a pulsed beam exhibits a broadening effect along its direction of propagation. Each pulse of light contains wave components that have a range of frequencies (colours), and, as a pulse moves through a material, these components separate - a process called dispersion. There are two types of dispersion: normal and anomalous. In normal dispersion, the low frequencies move faster than the high frequencies ('red leads blue'), whereas in anomalous dispersion, the high frequencies lead the low frequencies ('blue leads red').

However, a high-power beam can cause the change in the material's refractive index to shift The work will allow fundamental properties the lower frequencies ('red') to the front of the pulse and the higher frequencies ('blue') to the rear. By tuning the beam intensity, the effect of anomalous dispersion can be cancelled out. Furthermore, if a bright beam is turned off and then back on (a dark pulse), the frequency shifts are reversed and normal dispersion can be neutralized.

A $3 \mathrm{D}$ soliton, sometimes referred to as a light bullet, is the result of cancelling out diffraction and dispersion simultaneously (Fig. 1). Although such objects exist in theory, they are notoriously unstable. The focusing of the beam by the material must perfectly balance diffraction, and it is extremely difficult to counteract diffraction and dispersion at the same time, because these actions require different beam intensities. Scientists have generated solitons that are stable in two dimensions (one along the direction of propagation and one perpendicular to this direction $)^{3}$, and 3D solitons in a highly structured material (glass patterned with an array of optical devices called waveguides) ${ }^{4}$. But it has not been possible to create $3 \mathrm{D}$ solitons in an unstructured these objects and for practical applications.

It has been known for more than 20 years that self-guided light beams can be generated in photorefractive materials ${ }^{5}$. These are materials that exhibit a temporary change in refractive index when exposed to a beam of light, as a result of electrons moving through them. The focusing of the beam occurs in such a way that the need for perfect control of the beam intensity is eliminated. Furthermore, bound electrons produce the frequency-shifting refractive index required to eliminate dispersion. There is only one hitch: the material needs to have regions of negative and positive electric charge, but such a charge distribution takes time to establish - longer than the duration of a short light pulse.

Lahav and colleagues' solution was to shine a repetitive string of such pulses into a crystal of the photorefractive material strontium barium niobate, which responded to the power averaged over many pulses to create a self-guided beam. The response of the bound electrons in the crystal then allowed dispersion to be cancelled out in each pulse. The result was a string of 3D 'pulse-train' solitons - so named because the properties of each soliton depend on the solitons that come before it.

The authors used pulses of 800-nanometre wavelength, which meant that the crystal had normal dispersion. As a consequence, although the beam produced was bright, its temporal material - which is desirable for studying

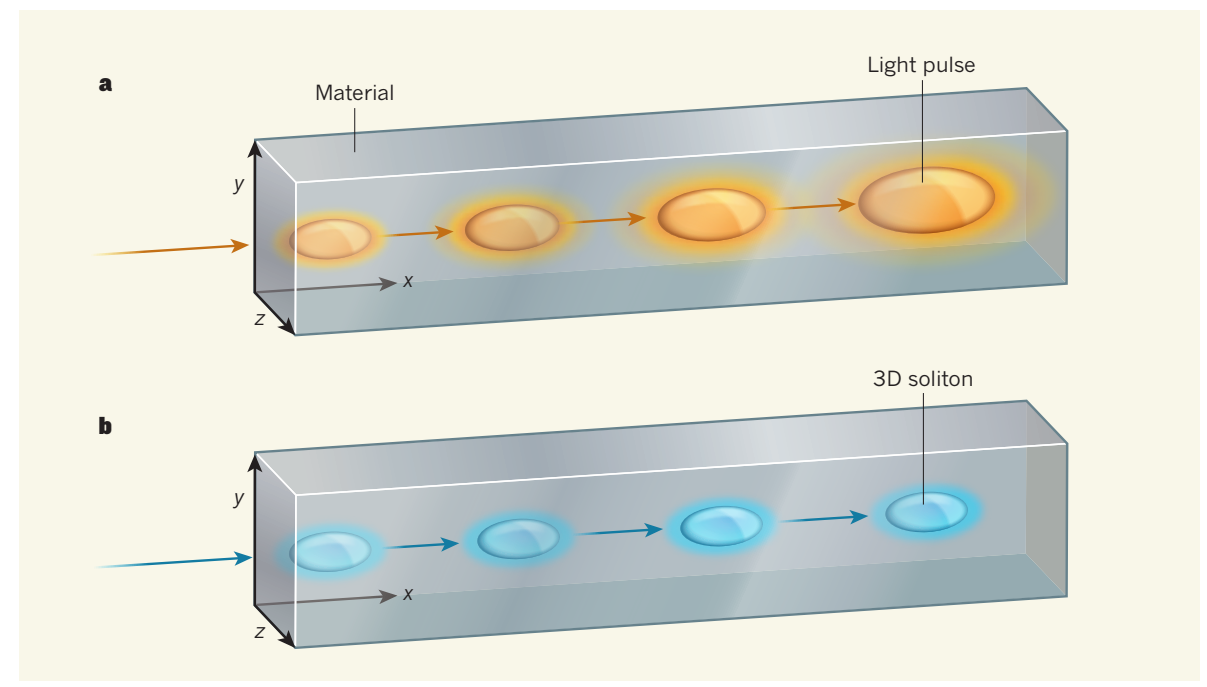

Figure 1 | Light pulses versus 3D solitons. a, A pulse of light tends to spread out as it propagates through a material (coloured arrows). It broadens along the direction in which it is travelling (the $x$ axis) as a result of dispersion, whereby components of the pulse that have different frequencies separate. Furthermore, it widens along the perpendicular directions (the $y$ and $z$ axes) because of diffraction. $\mathbf{b}$, Lahav et al. ${ }^{2}$ report a technique for producing three-dimensional solitons - packets of light that maintain their geometry as they move through a material. 


\section{RESEARCH NEWS \& VIEWS}

profile consisted of a dark pulse (see Figure 2 of the paper ${ }^{2}$ ). By performing similar experiments at longer wavelengths, for which the crystal has anomalous dispersion, it should be possible to generate bright 3D solitons - one of the major goals in the field of nonlinear optics.

Considering the difficulty in controlling localized 3D wave packets, Lahav and colleagues' results constitute a substantial advance. Interest in localized but non-spreading 3D wave packets extends well beyond optics, to areas as disparate as exotic states of matter known as Bose-Einstein condensates ${ }^{6}$ and excitations of substances called ferromagnetic colloids ${ }^{7}$. Furthermore, it should now be possible to investigate how 3D solitons interact when they collide - do they pass right through each other, interact or merge? This information might be useful some day for optical information processing ${ }^{8}$.

Frank W. Wise is in the School of Applied and Engineering Physics, Cornell University, Ithaca, New York 14853, USA. e-mail:frank.wise@cornell.edu

1. Kivshar, Y. S. \& Agrawal, G. Optical Solitons: From Fibers to Photonic Crystals (Academic, 2003).

2. Lahav, O. et al. Phys. Rev. X 7, 041051 (2017).

3. Liu, X., Qian, J. \& Wise, F. W. Phys. Rev. Lett. 82, 4631-4634 (1999).

4. Minardi, S. et al. Phys. Rev. Lett. 105, 263901 (2010).

5. Shih, M. et al. Opt. Lett. 21, 324-326 (1996).

6. Strecker, K. E., Partridge, G. B., Truscott, A. G. \& Hulet, R. G. Nature 417, 150-153 (2002)

7. Ackerman, P. J. \& Smalyukh, I. I. Nature Mater. 16, 426-432 (2017).

8. McLeod, R., Wagner, K. \& Blair, S. Phys. Rev. A 52, 3254-3278 (1995). 